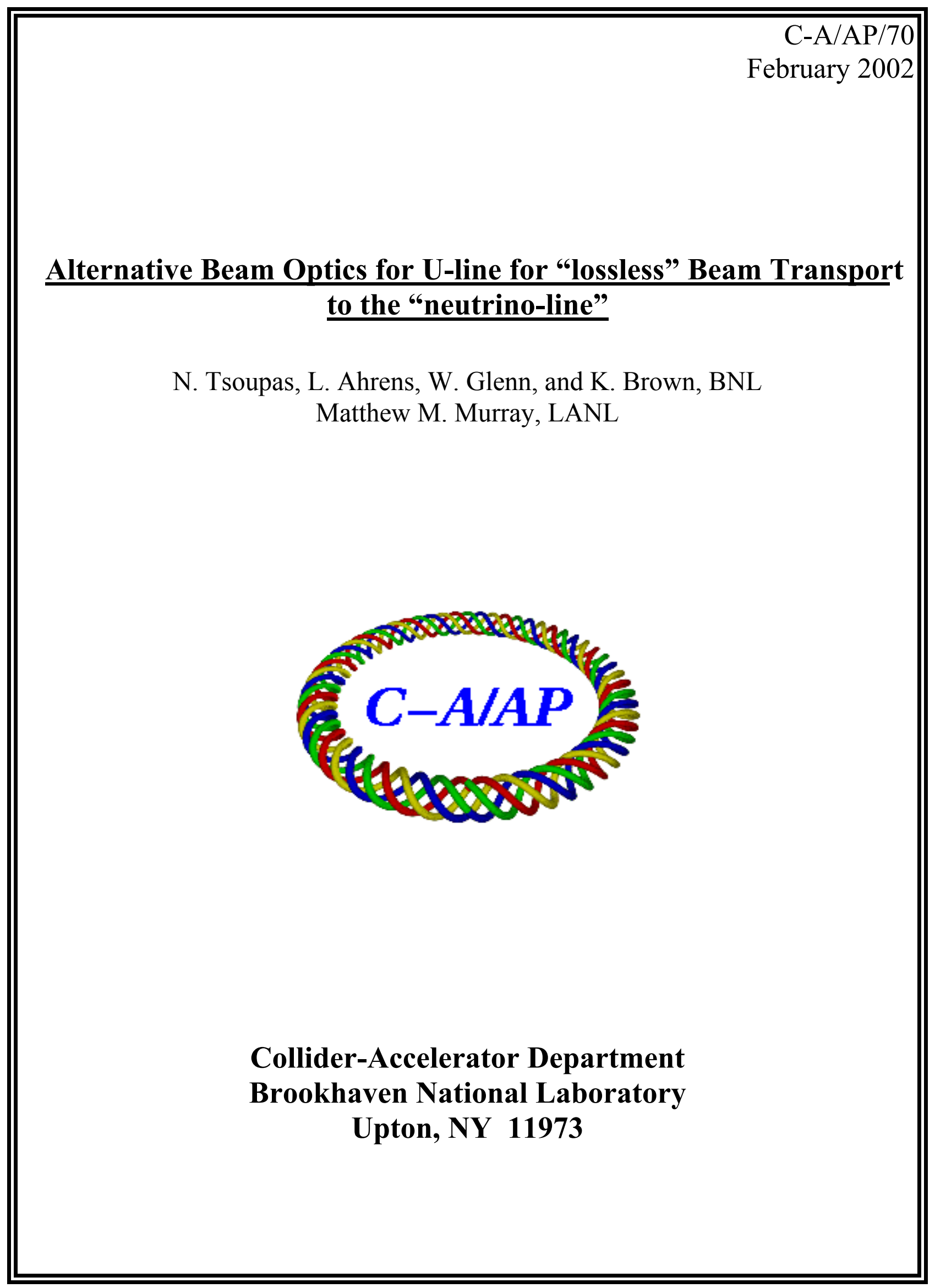




\title{
Alternative Beam Optics for U-line for "lossless" Beam Transport to the "neutrino-line"
}

\author{
N. Tsoupas, L. Ahrens, W. Glenn, and K. Brown BNL \\ Matthew M. Murray LANL
}

\begin{abstract}
The U-transport line ${ }^{1}[1,2,3,4]$ (shown as a Blue trace in figure 1) and its extension (the former "neutrino line" shown as red trace on Figure 1), are being used to transport the fast extracted[5] proton beam from the AGS synchrotron to various experiments which are located along the "neutrino line".

The beam transmission of the transport lines mentioned above, is limited by the vertical aperture of the combined function magnet WD1 whose location is shown in Fig. 1, The WD1 is the first magnet of the W-line ${ }^{2}[1,2,3,4]$ (shown as a Green trace in Figure 1) and is turned off during the beam transport to the experiments of the "neutrino-line" but it cannot be removed physically from the transport line since WD1 is part of the AtR transfer line.

There are indications, based on the beam profile at the location of the "visual-flag" "UF770" which is downstream of WD1 magnet, that the vertical edges of the beam are "clipped".

In order to improve the beam transmission at the location of the WD1 magnet we propose an alternative beam optics which minimizes the vertical beam size at the location of the WD1 magnet and at the same time satisfy the necessary constraints of the beam transport to the experiments of the "neutrino line".

In this technical note we describe the current beam optics for transporting the beam down to the Radiography experiment, and the "alternative beam optics" which minimizes the vertical beam size at the location of the WD1 magnet.
\end{abstract}

\section{Introduction}

The U-line and its straight-line extension "neutrino-line" which are shown in Fig. 1, have been used to transport Fast Extracted Beam (FEB) of protons from the AGS to various experiments which were located along the "neutrino-line". Few of the experiments that were performed on the "neutrino-line" were:

a) the "proton-Radiography" experiment,

b) the mercury-target experiment of the Spallation Neutron Source (SNS) and

c) material-testing experiments for the Accelerator Production of Tritium (APT) project .

The experiments utilized proton bunches with $\sim 10^{11}$ protons/bunch at a proton energy within the range of $\sim 2 \mathrm{GeV}$ to $\sim 24 \mathrm{GeV}$.

The beam transport to the "neutino-line" experiments was done using the existing magnetic elements of the U-line[1,2,3,4] and three additional quadrupole elements, UQ14, UQ15, and UQ16 (see Fig. 1) which are placed downstream of the WD1 dipole magnet (see figure 1) which is the first magnetic element of the W-line.

In order to keep the polarities of all the magnetic elements of the U-line the same as the polarities required during beam transport to RHIC, we tailored the beam transport optics to the "neutrinoline" experiments, on the "same-polarities" constraint.

\footnotetext{
${ }^{1}$ The U-transport line is the first section of the AtR beam transport line.

${ }^{2}$ The $\mathrm{W}$-transport line is the second section of the AtR beam transport line.
} 
The beam optics for the beam transport to the radiography experiment performed on the year 2000 is presented in the next section.

\section{Original Beam Optics for the Radiography Experiment performed in the "U-line-neutrino_line" the year 2000}

The beam transport to all of the "neutrino-line" experiments was performed by keeping the polarities of the U-line quadrupoles identical to the polarities used for the beam transport into the RHIC machine.

The "same polarity" constraint was intentional in order to minimize the time required to switch from the "neutrino-line" experiment to RHIC beam-injection operations and vise-versa.

An additional constraint imposed by the "neutrino-line" experiments is also the beam-size on the target of the experiment.

The "radius" of the beam-size on the experimental target varied from $\sim 1 \mathrm{~mm}$ to $\sim 10 \mathrm{~mm}$ depending on the requirements of the experiment. This "radius" corresponds to one standard deviation of the beam intensity distribution. These two constraints ("same-polarity" and "beamsize on target") impose a lower limit on the vertical beam size at the location of the WD1 magnet. For the Radiography experiment, the standard deviation $\left(\sigma_{\mathrm{x}, \mathrm{y}}\right)$ of the beam distribution on the target ("diffuser") was $1 \mathrm{~mm}$. The corresponding horizontal and vertical beam profiles which contain one standard deviation $\left(1 \sigma_{\mathrm{x}, \mathrm{y}}\right)$ of the beam intensity along the U-line are shown in figure 2. In the calculations of the beam profiles, shown in figures 2 and 7 (see next section), we assumed a normalized beam-emittance of $40 \pi \mathrm{mm}$.mrad that contains $90 \%$ of the beam distribution. The beam momentum is $\sim 25.6 \mathrm{GeV} / \mathrm{c}$.

In particular the maximum size of the standard deviation of the vertical beam profile, at the location of the WD1 magnet, is $\sigma_{y}=5 \mathrm{~mm}$ and is at the exit of the WD1 magnet as shown in figure 2 , and the corresponding horizontal beam size at the same location (exit of WD1) is $\sigma_{x}=1.5 \mathrm{~mm}$. The beam profile at the location of the visual flag "UF770" which is located $\sim 7 \mathrm{~m}$ downstream of the WD1 magnet indicates that the beam is scrapped vertically by the WD1 magnet.

A comparison of the beam size at the exit of the WD1 magnet with the available aperture at the same location may be a convincing argument that an alternative beam optics that will minimize the vertical beam size at this location (exit of WD1) maybe desirable. This comparison is presented in the rest of this section.

Figure 3 shows a cross section of the WD1 magnet looking upstream, with some relevant dimensions. The WD1 magnet is a combined function ${ }^{3}$ magnet therefore the pole faces of the magnet are not parallel.

Figure 4 shows a drawing of the cross section of the WD1 magnet, also looking upstream, with the vacuum chamber cross section at the entrance of the magnet (the oval shaped line traced in blue) and at the exit of the magnet (the oval shaped line traced in blue at the left of the vertical symmetry axis and traced in red at the right of the vertical symmetry axis).

Figure 5 is a drawing of the cross section of the chamber at the entrance of the magnet (gray oval shape which is symmetric with respect to vertical symmetry axis) and at the exit of the magnet (non-symmetric oval shape, gray in color at the left of the symmetry axis, and red in color at the right of the symmetry axis). The dimensions on the drawing are in inches.

Figure 6 is a top view of the trajectories of the central ray in the WD1 magnet which is a $3.658 \mathrm{~m}$ long. The red trace corresponds to the non-deflected beam which is directed into the "neutrinoline", and the green trace corresponds to the beam directed into the W-line. At the exit of the magnet, the central ray of the non-deflected beam (red-trace) is located $6.4 \mathrm{~cm}$ to the right of the

\footnotetext{
${ }^{3}$ It provides both, a dipole field for bending, and a quadrupole field for focussing.
} 
symmetry axis (looking upstream) of the chamber. At this location the available vertical aperture of the WD1 magnet is $\sim \pm 15 \mathrm{~mm}$, and horizontal aperture is $27 \mathrm{~mm}$ from the beam center and to the left side of the beam direction. On the right side of the beam direction the aperture may be considered as "infinite".

Table I tabulates the horizontal and vertical beam sizes and available apertures at the exit of the WD1 magnet for the two beam transports we discuss in this technical note.

\begin{tabular}{|c|c|c|c|c|c|c|}
\hline & \multicolumn{2}{|c|}{ Beam size $[\mathrm{mm}]$} & \multicolumn{2}{c|}{ Available aperture $[\mathrm{mm}]$} & \multicolumn{2}{c|}{ \# of available $\sigma^{, \mathrm{s}}$} \\
\hline & $\sigma_{\mathrm{x}}$ & $\sigma_{\mathrm{y}}$ & Horizontal & Vertical & Horizontal & Vertical \\
\hline Original Optics & 1.5 & 5.0 & $+27,-\infty$ & \pm 15 & 18.0 & 3.0 \\
\hline Alternative Optics & 3.7 & 1.0 & $+27,-\infty$ & \pm 15 & 7.3 & 15.0 \\
\hline
\end{tabular}

TABLE I: Horizontal and Vertical beam sizes (columns 2,3) at the exit of WD1 magnet for two beam-transports (column 1). The available Horizontal and vertical apertures of the magnet is shown in columns 4,5 .

The "beam-clipping" shown by the "UF770" flag prompted us to try an alternative beam transport optics. In the next section we discuss the alternative beam transport optics in which the vertical beam profile is minimized at the location of the WD1 dipole magnet.

\section{Alternative Beam Optics of the "U_line-neutrino_line" for minimum vertical beam size at the location of WD1.}

In this alternative beam optics of the "U-line-neutrino_line" the physical locations of the dipoles and quadrupole magnets ${ }^{4}$ are kept the same as in the beam optics discussed in the previous section. However the polarity of specific quadrupoles (UQ11, UQ12, UQ13) of the Uline is reversed.

Figure 7 shows the beam profiles of the alternative optics for beam transport to the target of the radiography experiment.

The location of the magnets of the alternative optics shown in figure 7 is identical to that of the magnets shown in figure 2 , with the only difference between the optics corresponding to the figure 2 and the optics corresponding to the figure 7 being the polarity of the UQ11, UQ12, and UQ13 quadrupoles and the strength of the quadrupoles.

The "alternative optics" shown in figure 7 provides a small vertical beam size $\left(\sigma_{x}=1 \mathrm{~mm}\right)$ at the location of the WD1 magnet as compared to the vertical beam size $\left(\sigma_{\mathrm{x}}=4.9 \mathrm{~mm}\right)$ at the same location shown in figure 2 . However the horizontal beam size has a standard deviation $\sigma_{\mathrm{x}}=3.7 \mathrm{~cm}$ Table I summarizes the beam sizes and apertures at the exit of the WD1 magnet.

Due to limitations on the upper limit strength of the last two quads (UQ15 and UQ16) of the transport line, the beam size at the target location cannot reach the $\sigma_{\mathrm{x}}=1 \mathrm{~mm}$ size as in the case shown in figure 2. This however can be remedied by placing the target (Diffuser) of the Radiography experiment $2 \mathrm{~m}$ downstream of the present location where the beam can be focused to a beam size with $\sigma_{\mathrm{x}}=1 \mathrm{~mm}$.

\footnotetext{
${ }^{4}$ The quadrupoles in the figures 2 and 7 are shown as thin rectangular boxes positioned above the $\mathrm{x}$-axis (focusing quads) or below the $\mathrm{x}$-axis (defocusing quads). The wider rectangles shown on the same figures represent the dipoles.
} 


\section{Conclusion}

By inverting the polarity of the quadrupoles (UQ11, UQ12, UQ13) of the U-line we can adjust the optics of the transfer line and provide better beam transmission to the various experiments located on the "neutrino line". Under the present conditions the polarity inversion of the quadrupoles requires at least $1 / 2$ an hour per quadrupole. The polarity inversion therefore may present a problem in case the experiments are performed in parallel with the RHIC operations. The experimental knowledge of the beam parameters at the beginning of the U-line will provide a more accurate beam transport in the "U_line-neutrino_line"; therefore it is recommended that measurements of the beam emittance and the beam parameters at the beginning of the U-line are performed. These measurements can be accomplished by using the method described in ref. [6].

\section{REFERANCES}

[1] Focusing and Matching Properties of the AtR Transfer Line

N. Tsoupas et. al. BNL,

PAC97 Vancouver, BC, May 12-16 (1997)

[2] AGS to RHIC Transfer Line: Design and Commissioning W.W. MacKay et.al. BNL, European Particle Accelerator Conference 1996 Barcelona Spain (RHIC/AP/103)

[3] Physics of the AGS to RHIC Transfer Line Commissioning L. Ahrens et. al. BNL, European Particle Accelerator Conference 1996 Barcelona Spain

[4] "Beam Transfer from AGS to RHIC" J. Claus and H. Foelsche RHIC-47, 1988

[5] "Fast Extracted Beam for the g-2 Experiment" N. Tsoupas et. al. C-A/AP/54 June 2001

[6] "A computer code to measure the beam emittance and beam parametrs of the AtR Line" N. Tsoupas et. al. C-A/AP/42 March 2001 


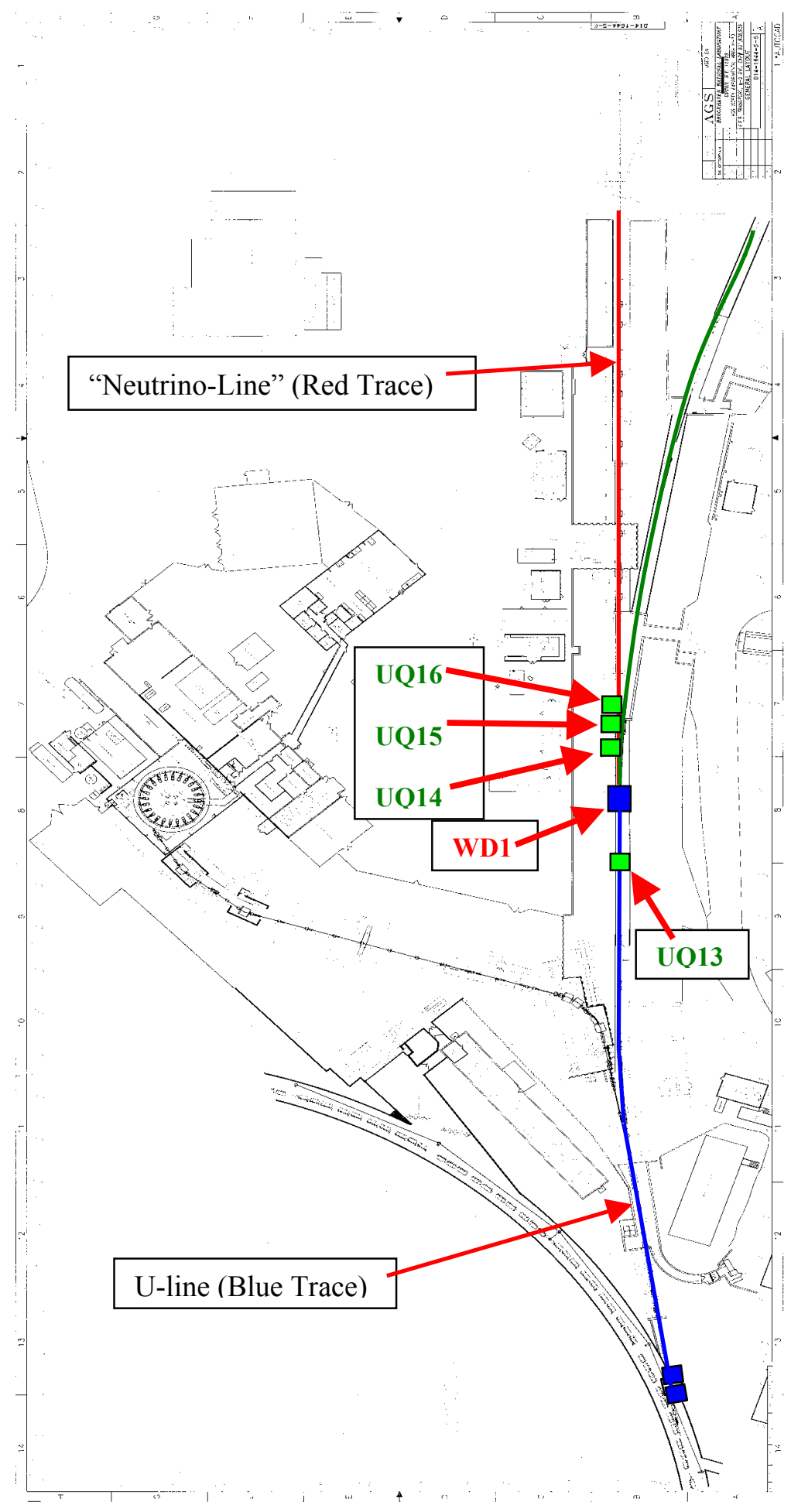

Figure 1 Schematic diagram of the U-line, (Blue Trace) "Nuetrino-line, (Red Trace) and W-line (Green Trace) showing the location of the WD1 magnet. 


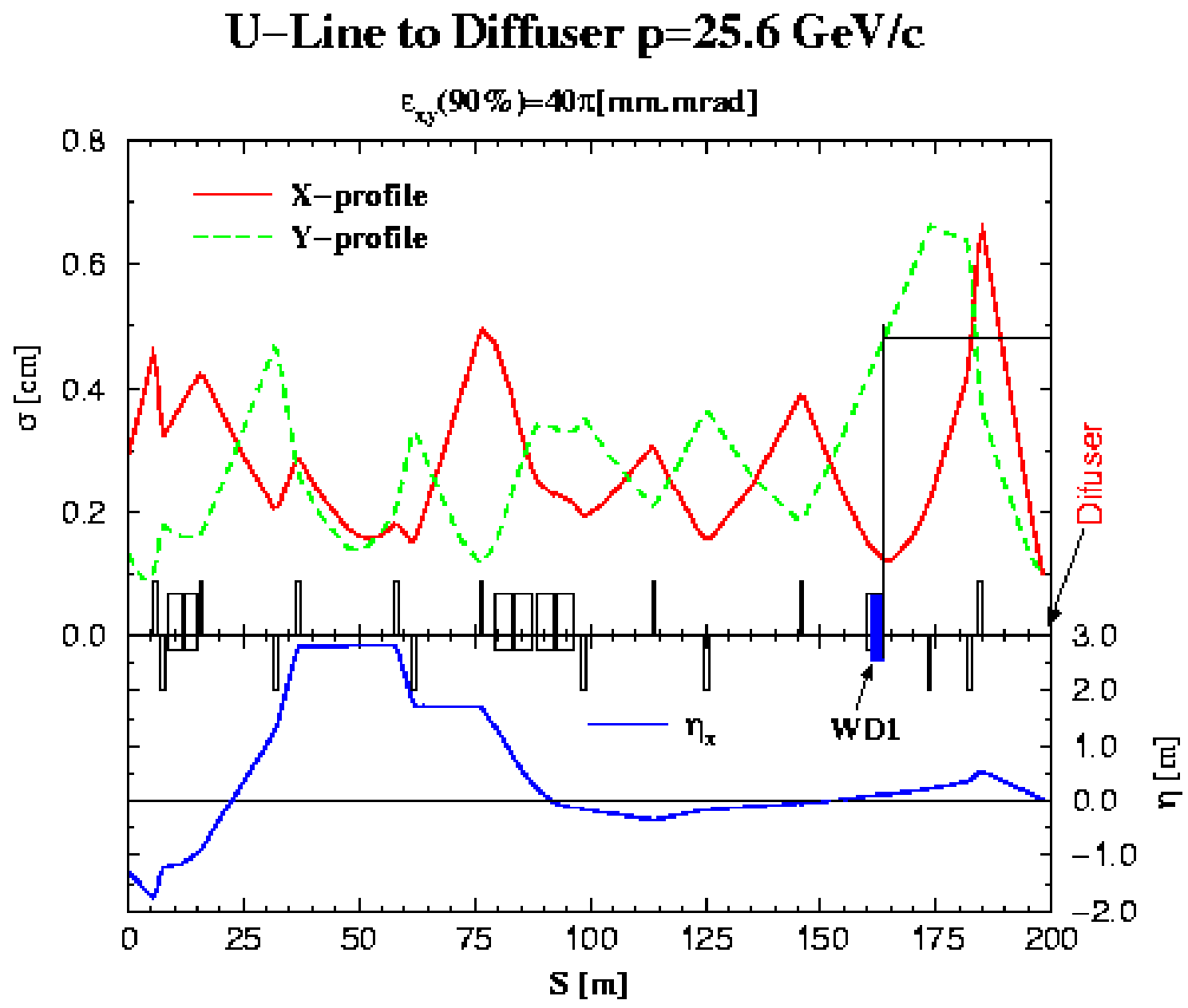

Figure 2. The Horizontal and vertical sizes of the standard deviations of the beam distribution along the "U_line-Neutrino_line". The Horizontal and Vertical beam emittance was assumed $40 \pi . \mathrm{mm} . \mathrm{mrad}$ and the beam momentum $25.6 \mathrm{GeV} / \mathrm{c}$ 


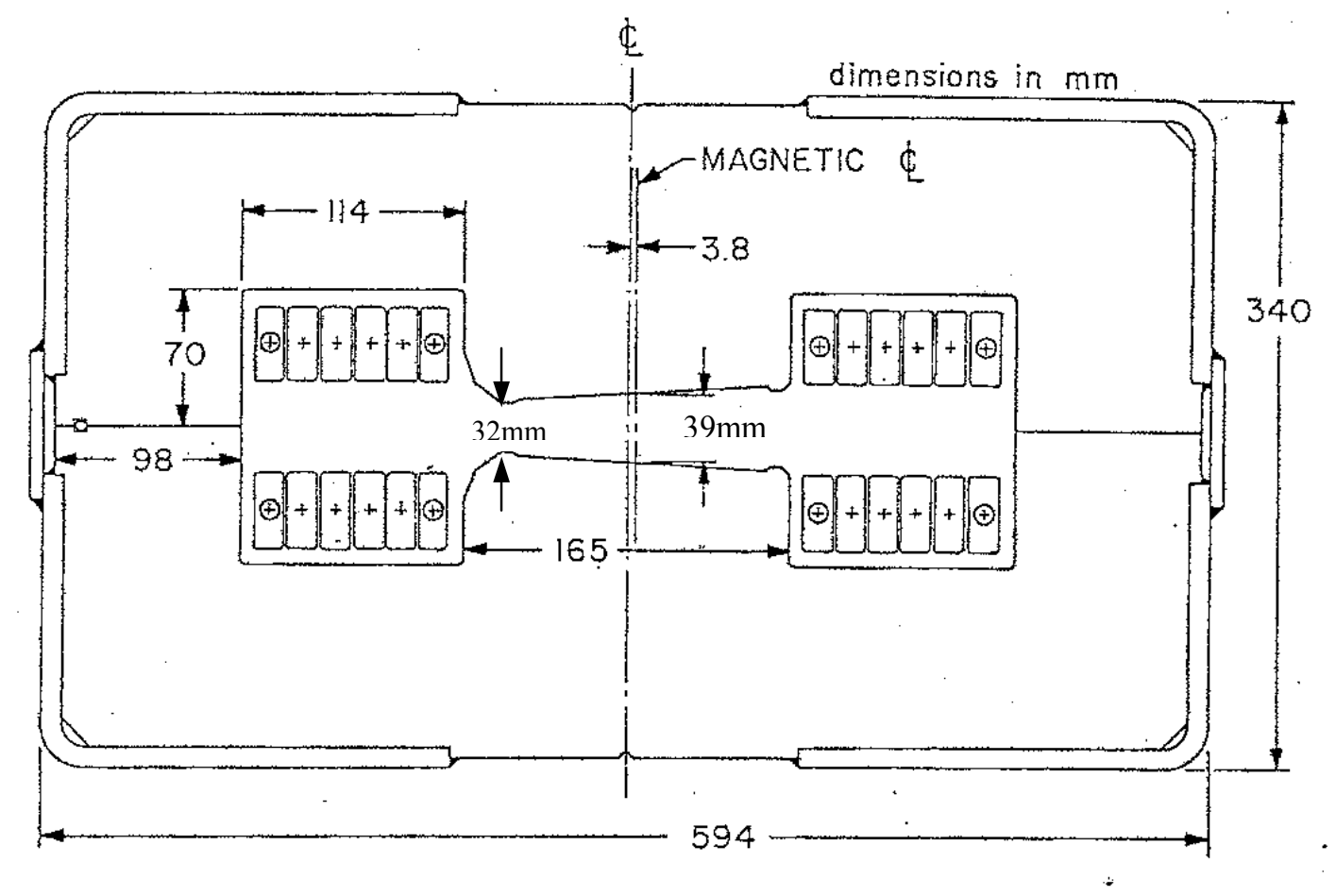

Figure 3. Cross section of the WD1 combined function magnet, as it appears looking upstream. 


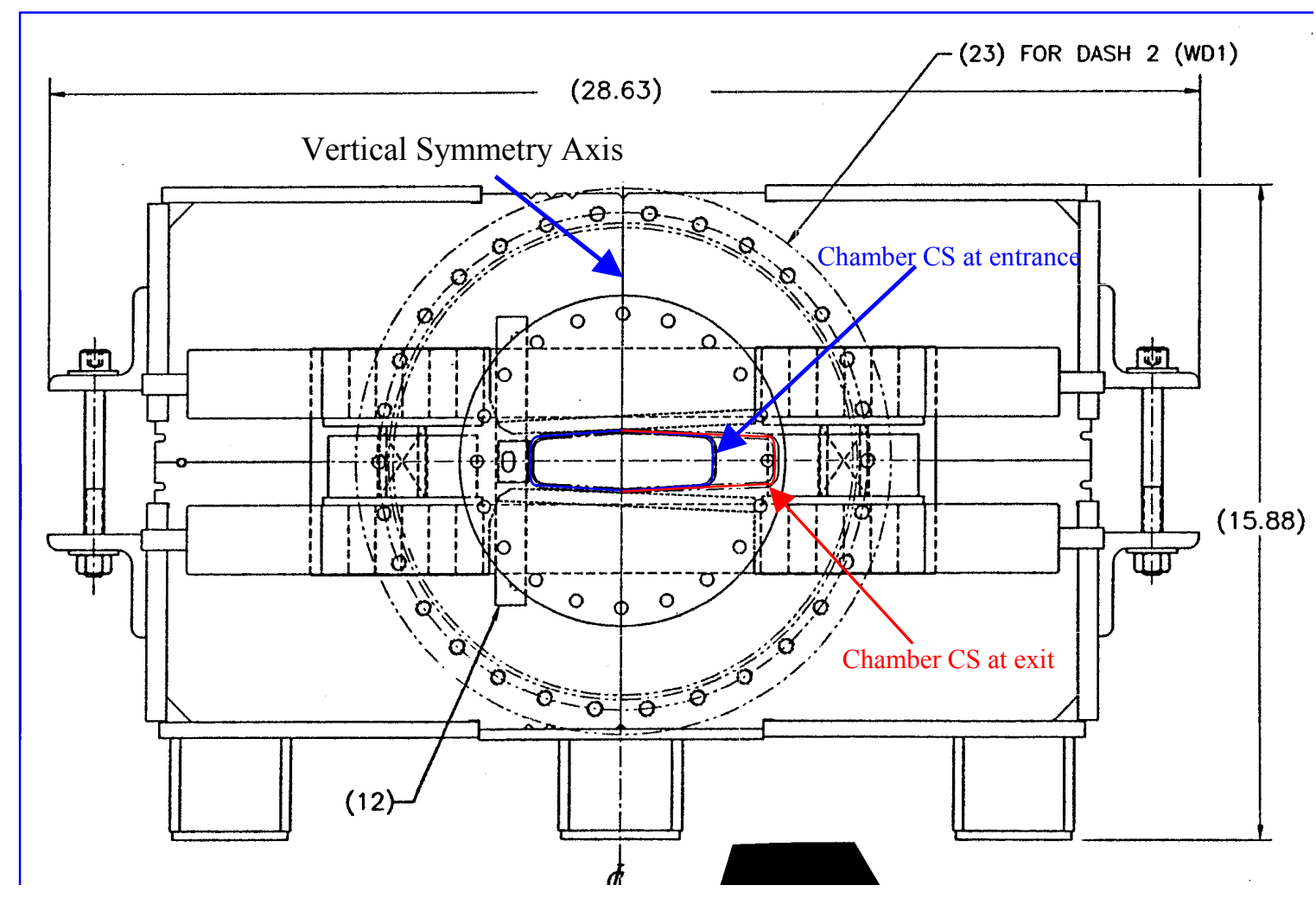

Figure 4. Drawing of the cross section of the WD1 combined function magnet, as it appears looking upstream. The cross section of the vacuum chambers at the entrance of the magnet is drawn with a blue line, and is symmetric with respect to the vertical symmetry axis. The cross section of the vacuum chamber at the exit of the magnet and on the left of the vertical symmetry axis is the same with the cross section of the chamber at the entrance, but on the right of the symmetry axis the chamber is wider (red trace) to accommodate the transported beam into the "neutrino-line". 


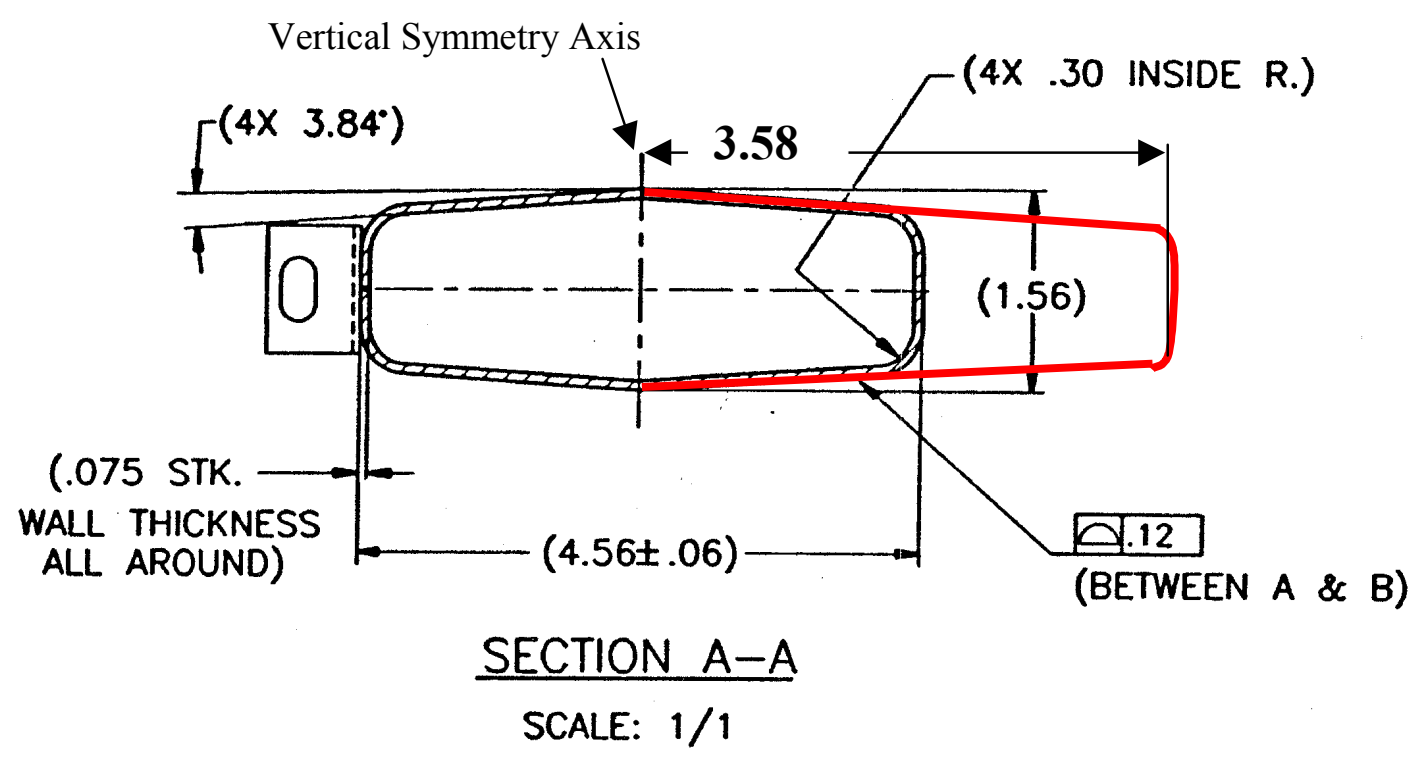

Figure 5. Drawing of the cross section of the vacuum chamber of the WD1 combined function magnet, as it appears looking upstream.

The cross section of the vacuum chambers at the entrance of the magnet is drawn with a grey line, and is symmetric with respect to the vertical symmetry axis. The cross section of the vacuum chamber at the exit and on the left of the vertical symmetry axis is the same with that of the entrance, but on the right of the symmetry axis the vacuum chamber is wider than it is on the left (red trace) in order to accommodate the transported beam into the "neutrino-line". 


\section{Beam to the Radiography Experiment}

Vertical available gap at beam-exit= $32 \mathrm{~nm}$

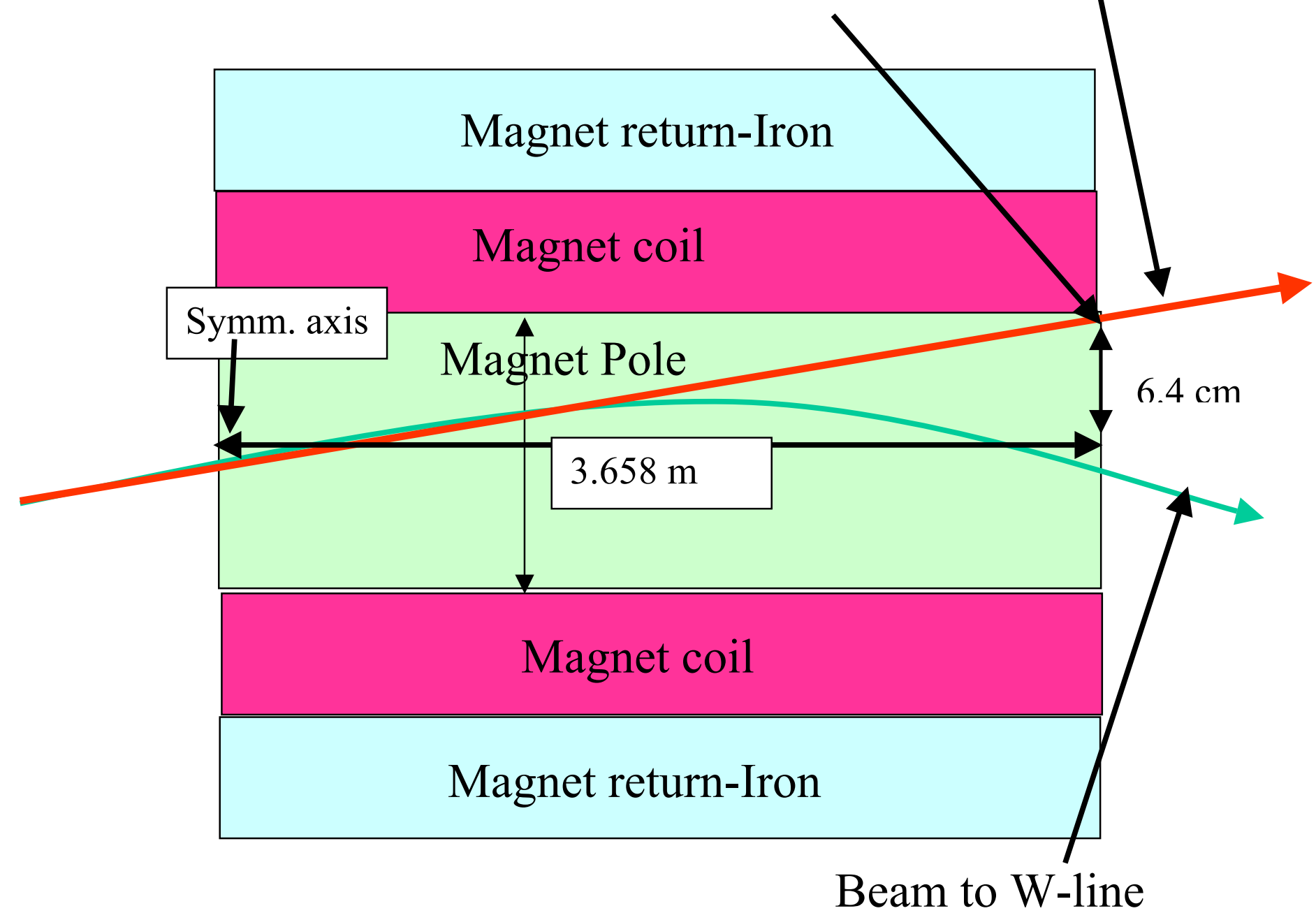

Figure 6. Top view of the central beam trajectories at the median plane of the WD1 magnet. The green trace corresponds to the central beam trajectory of the W-line, and the red trace to the central beam trajectory of the "neutrino line". 


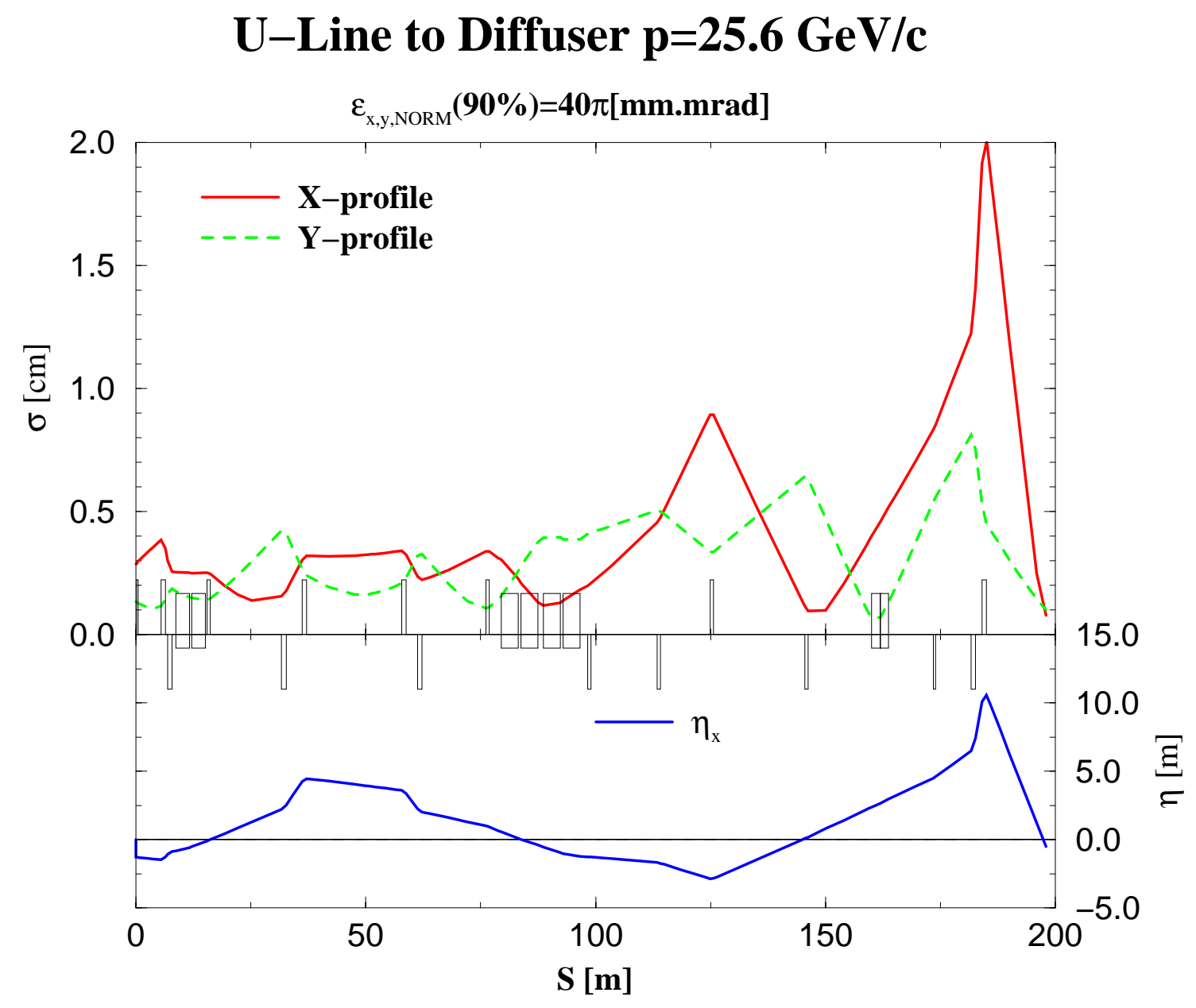

Figure 7. The Horizontal and vertical sizes of the standard deviation of the beam distribution along the "U_line-Neutrino_line" for the alternative beam transport optics. The Horizontal and Vertical beam emittances each was assumed $40 \pi$.mm.mrad and the beam momentum $25.6 \mathrm{GeV} / \mathrm{c}$ 